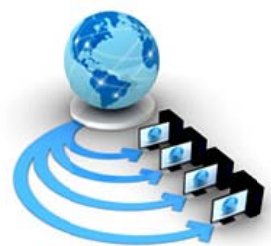

Volume 9, No. 1, January-February 2018

International Journal of Advanced Research in Computer Science

REVIEW ARTICLE

Available Online at www.ijarcs.info

\title{
DYNAMIC SUBSTANCE BASED PICTURE CHASE AND RECUPERATION BY JOINING LOW LEVEL FEATURES
}

\author{
Vahini siruvoru \\ Assistant Professor in CSE \\ S R Engineering College \\ Warangal, Telangana, India
}

\author{
Nampally vijay kumar \\ Assistant Professor in CSE \\ S R Engineering College \\ Warangal, Telangana, India
}

\author{
Soumya Sathuri \\ PG Research Scholar \\ S R Engineering College \\ Warangal, Telengana, India
}

\begin{abstract}
With the Advancements and noticeable quality of the casual association and enhancement of blended media development, the ordinary Image recuperation don't mollify the wants of customer. In current days with the development of long range relational correspondence mediums, such countless pictures are exchanged well ordered. Remembering the true objective to get to this broad picture amassing the new methods are amazingly urgent. Picture mining structure is the Content-Based Image Retrieval (CBIR) which completes recuperation in perspective of the comparability portrayed similar to removed features with more goodness. In this paper, dynamic substance based picture request and recuperation is given, called Hybrid component extraction method. The discernable substance of a photo, for instance, shape, shading and surface are profited in content-Based Image Retrieval (CBIR).The Proposed figuring which relate the upsides of specific estimations to upgrade the execution and precision of recuperation. The precision of shading histogram based organizing can be expanded by using Color Coherence Vector for dynamic depuration. The Fourier Descriptors with Fast Fourier change and Extended Hough change can strengthen the speed of shape based recuperation. The Gabor channel has been all things considered grasped to isolate picture features, especially surface features. Feature Vector Normalization can be set to guarantee that unmistakable component vectors in the similarity estimation process. Thusly the surface, shading and shape features are joined to give a prosperous rundown of capacities to recuperating picture. Additionally, essentialness input (RF) plot is refined to propel the achievement of substance based picture recuperation (CBIR).
\end{abstract}

Keywords: Content-Based Image Retrieval, Interactive Multimedia Processing, Biased Discriminant Analysis, Relevance Feedback, Machine Learning, Wavelet, Gabor filter and Bayesian network.

\section{I.INTRODUCTION}

Degrees of advance in picture fulfillment and cutoff headway have incited surprising change in incredibly colossal picture databases. in the event that analyzed[1], These photographs can indicate consummate data to the human clients. There are three requests of picture recovery methodology: content based, content-based, and semantic based. In content based frameworks, the photographs are kind remarked by content descriptors. Content illumination to all photographs masculine is unattainable in light of expansive stamping cost and the common of human acknowledgment. To toppled the above obstruction in content based picture recovery structure, Content-Based Image Retrieval (CBIR)[2] was uncovered .CBIR depends upon indubitably asking for and recovery and It plans to search for pictures that are perceptually like the request picture in context of prominent substance of the photographs without the assistance of comments. Masters generously revolve around the capable low-level delineation of pictures and CBIR by and large records pictures by low-level visual highlights. Content Based Image Retrieval (CBIR) is a revile undertaking. Routinely[3], In CBIR framework the photograph highlights are in three classes: shading, surface and shape and these highlights ought to be blended to serve better inclination in the relationship philosophy. Shading is a definitive ordinary fragment utilized as a bit of CBIR, particularly due to getting shading data from pictures. Secluding information around shape and surface part are more caught and expensive undertakings, by and large performed after the soonest separating given by shading highlights[4]. The Image highlights which can be named bizarre state and low-level highlights. Clients would ask for have the ability to depiction pictures in context of these lowlevel highlights. By closeness count our pined for picture from the photograph record is gotten. The key issue of CBIR is the contrast between the sporadic state semantic musings utilized by people to see picture content and the low-level recognizable highlights got from pictures utilized by a PC to list the photographs in a database. Two most preposterous critical research fields in CBIR are the picked of the utilized highlights, the level of character among highlights and the procedure for asking for the photographs ie., how to pick the photographs the structure will show to the client next. Normal applications remain requiring clear frameworks for looking of pictures in light of their aggregate appearance. For instance, a client may demand to pass on all photographs unclear to a given request picture from a 
tremendous storeroom of pictures[5]. Roshi Choudhary prescribe shading histograms are appropriate outcome to this issue, the histogram portray the lessen level or shading development for a given picture, they are totally effective, yet ordinarily coldblooded to little collection in camera scène. There are two or three checks in shading histograms ie.,No spatial learning is directed by shading histogram and it exclusively delineate which tones are available in the photograph, and in what degree[6]. Furthermore, shading histograms are dubious to both weight old-fashioned rarities and changes with everything taken into account photograph chart; we require audit shading space, a shading quantization methodology, a histogram portrayal and a resemblance metric for the course of action of histogram based system. An electronic picture in this substance is a course of action of pixels and every pixel tends to a shading. Tones can be tended to utilizing unmistakable shading spaces relying on the models got a handle on by the experts or create on the application, for example, Red-Green-Blue (RGB), HueSaturation-Value (HSV), YIQ or YUV and whatnot. A shading based philosophy is used for separating pictures which are undefined with shading histograms, yet which moreover ponders spatial data. The general picture consolidate delineation approaches have been utilized as a bit of picture dealing with and contentbased picture recovery[7],[8],[9]. I) Grid shading minute ii) Local coordinated delineation (LBP) iii) Gabor wavelets surface iv) Edge introduction histogram. Cross section shading minute: The framework shading minutes are everything viewed as utilized for ousting shading highlights from pictures. Particularly, a photograph is separated into a $3 X 3$ cross segments. For every lattice, three sorts of shading minutes are secluded. They are shading mean, shading change, and shading skewness in each shading channel (R, $\mathrm{G}$, and B), only. Contiguous twofold case (LBP): The Local parallel case (LBP)[10] is depicted as a dull scale invariant surface measure, got from a general significance of surface in a territory. Gabor wavelets surface: An Image is extended to 64 X64 pixels to evacuate Gabor surface highlights, and a brief span later apply the Gabor wavelet change. Edge introduction histogram: An edge introduction histogram is expelled for every photograph, at first every photograph is changed over into a grayscale picture then attentive edge marker is figured out how to accomplish the edge outline deciding the edge introduction histogram[11],[12].

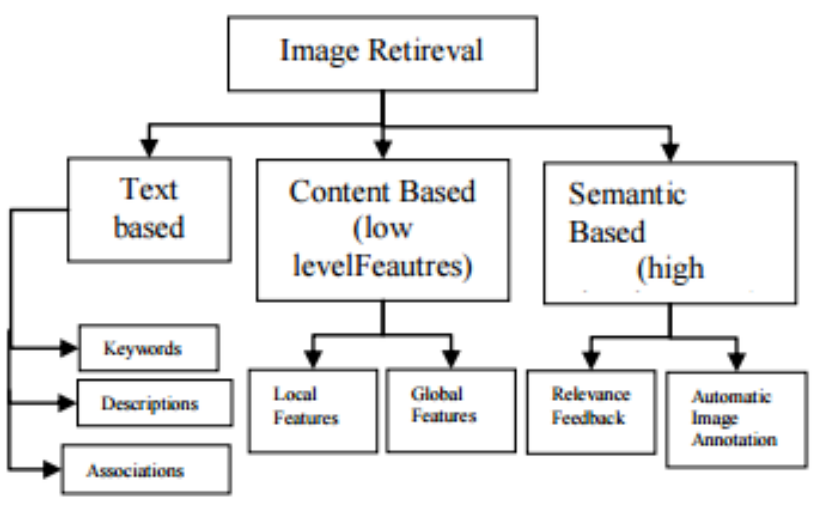

Figure 1.Image retrieval categories.

In this way, picture recovery can be depicted as the task of filtering for pictures in aimage database. CBIR frameworks look social affair of pictures [13] in setting of highlights that can be isolated from reports of imagine themselves without unmistakable. The objective of CBIR structures is to sponsorship picture recovery in light of substance e.g., figure, shading, surface[14]. In past decades diverse CBIR structures have been passed on, the common conviction for them is to remove a pined for picture. Isolating two pictures and picking if they are practically identical or not is a fairly fundamental thing to achieve for a human. Getting a PC to do in like way effectively is however a substitute issue. The considers used a touch of these procedures are dependably pulled back into three errands: extraction, social occasion and confirmation. The extraction mission is to changes over well off substance of pictures into various substance highlights. Extraction of highlight is the arrangement of making portions to be used as a touch of the choice and game-plan assignments. Highlight choice decreases the measure of highlights suited connection task. Those makes which are inclined to help in slant are picked and used as a touch of the demand assignment. Highlights which are not picked are disposed of. Of these three activities, extraction of highlight is most important on the crucial that the particular highlights made open for partition particularly impact the sufficiency of demand undertaking. The last consequence of the extraction undertaking is a system of segments, consistently called highlight vector, which constitutes picture delineation. In the latest couple of decades, various above revealed frameworks of picture substance feature extraction sufficiently requested strong for helpful uses in mechanical mechanization, regulated hold reserves, biomedicine, awful conduct avoidance and biometric affirmation[15].

\section{RELATED WORK}

Our work is identified with three basic social affairs of research: content-based picture recovery, separate metric learning, and internet learning. In the running with, we quickly contemplate the relentlessly related master works in every get-together.

A. Content-Based Image Retrieval: With the expedient change of forefront cameras and photograph sharing areas, picture recovery has wound up being a champion among the most key research centers in the prior decades, among which content-based picture recovery is one of key testing issues.[2] The target of CBIR is to look pictures by reviewing the bona fide substance of the photograph rather than examining metadata like catchphrases, title and creator, with the genuine target that wide endeavors have been improved the situation exploring assorted low-level segment descriptors for picture delineation. For instance, specialists have spent different years in center unmistakable general highlights for picture delineation, for example, shading highlights [14], edge highlights, and surface highlights. Late years besides witness the surge of research on near to part based delineation, for example, the sack of-words models utilizing neighborhood highlight descriptors. Standard CBIR approaches by and large pick undaunted separation wears down some expelled low level highlights for sight and sound closeness look, for example, the standard Euclideann division or cosine similarity. Regardless, there exists one key obstruction that the settled unyielding resemblance/disconnect point of confinement may not be 
constantly flawless in light of the inclination of visual picture delineation and the rule trial of the semantic opening between the low-level visual highlights expelled by PCs and bizarre state human affirmation and clarification. Accordingly, late years have witnesses a surge of dynamic research endeavors in plan of different segment/similarity measures on some low-level highlights by mauling machine learning procedures, among which several works spin around understanding how to hash for reduced codes and some others can be planned into remove metric finding that will be shown in the going with partition. Our work is in like way identified with multimodal/multiview analyzes, which have been extensively considered on picture depiction and test certification fields. Regardless, it is frequently difficult to manhandle these philosophies plainly on CBIR in light of the way that (I) everything considered, picture classes won't be given unequivocally on CBIR errands,[16] (ii) paying little regard to whether classes are given, the number will be sweeping, (iii) picture datasets have a tendency to be widely more prominent on CBIR than on strategy attempts. We along these lines avoid the snappy associations with such existing works in this paper. There are still some other open issues in CBIR mulls over, for example, the ability and flexibility of the recovery system that a significant part of the time requires a successful asking for design, which are out of this present paper's degree.

\section{III.CONTENT-BASED SEARCH}

Most existing P2P structures[15] give particularly constrained substance look limits, for instance, search for in context of record title, producer names, catchphrases, or illustrative substance. To recover the suitable substance all the more viably, we require an approach that gives wealthier searching for highlights. Content-based yield is key for tending to extract archives, and it is likewise charming for tending to natural media information when content illuminations are nonexistent or lacking. Mixed media content asking for and recovery has been a dynamic field for over 10 years. It draws huge research exertion from the canny world, the bleeding edge, and the standard affiliations. For instance, MPEG-7 is a standard supported by the International Organization for Standardization for depicting the canny media content. It offers help to a wide degree of vocations, and it will affect the web as accessible for media to content as it is open for content. The movement of the World Wide Web, including the presentation of Rich Site Syndication (RSS), Web 2.0, and the semantic web, draws in the web data be machine process capable (rather than being basically human organized), appropriately empowers programs or assorted directors to discover, offer and join data all the more sufficiently. In this segment we will quickly depict a few occasions of substance based demand in P2P frameworks.

Tang et al. [20] proposed Peer Search, a competent P2P data framework that sponsorships content and semantic pursue. Companion Search grows existing data recovery systems: the vector space show up (VSM) and the sit without moving semantic asking for (LSI) to work with the useful controlling portions in a Content Addressable Network (CAN). For the most part, LSI utilizes particular respect separating to change and truncate a framework of narrative vectors selected from VSM to find the semantics basic terms and records. The producers utilized the semantic vector of a record as the best way to deal with store the narrative archive in CAN, with a definitive target that the once-overs set away abutting in CAN are shut in semantics. A near advancement can be related with sound or video information, where the semantic vectors must be separated from sound or video information. Lu and Callan looked into content-based asset confirmation and document recovery calculations in cross breed P2P systems. In their approach, the leaf focus picks the recovery occurs for certain demand utilizing probabilistic data recovery check, and the document focus point (supernode) builds up an assembled substance show up for the vast majority of its leaf focus focuses and a strategy of neighboring registry focus focuses. The substance demonstrate is utilized for managing demand messages.[17] Yang delineated a substance based music recovery framework in $\mathrm{P} 2 \mathrm{P}$ condition. Every strong report is changed over into a surge of trademark groupings, where each movement is a vector tending to a short bit of music information. Every last trademark movement are recorded utilizing Locality-Sensitive Hashing plan, to such an extent, to the point that equivalent (in term of human intuition) vectors can be hashed into a near hash an inspiration with high likelihood. Given a request sound, the recovery strategy is to discover a quick overview of matches on trademark game-plans with the adaptability of beat changes. To overhaul the demand proficiency in a P2P space, a twoarrange look convention was proposed.[18] In the presearch arrange, the request peer bestows a little subset of demand vectors to every potential companion. In the veritable intrigue orchestrate, peers with a higher shot of a hit will lead the more comprehensive demand. In Lee and Guan demonstrated a substance based picture recovery framework over a P2P form. Each accomplice in the structure keeps up two research tables, one for non specific neighbors which are normally the neighbors with the base physical bob checks, and the social affair neighbors which share basic premiums at the photograph content. The framework utilizes the obvious recovery results to see the social event neighbors, to such an extent, to the point that the consequent recovery inside the get-together neighbors will happen better recovery precision. The got a handle on visual highlights join shading, shading minutes, question shape, and surface.

\section{IV.PROPOSED SOLUTION}

We introduce two additional concepts in offline phase of P2PCBIR which will help to achieve reduced search time.

\subsection{Clustering of Peers}

Partners must be collected in light of any parameters like accomplices in a specific land a zone. Each accomplice will advance the measure of asking for it has gotten till later to every last one of the neighbors in its property a zone with a parcel of K ricochets[16]. Right when every last one of the partners have traded the data, the associate focus with most phenomenal number of enthusiasm till now will change into the package head. The diverse accomplices will come the bit of the get-together. An accomplice focus point can likewise be a touch of two social events. This happens for the case of visitor peers. The pack head accomplice will begin a versatile executive. This adaptable director will visit every 
last one of the partners in the social event and visit the pack head. The adaptable director will pass on data of the photograph include package data in each accomplice to the social event head peer[19].

\subsection{Constructing Search Index for Peer Cluster}

The convenient administrator will bring the segment vector of pictures in each friend to the gathering head peer. Arranged in this grouping is again done to hoard the photo features. The compact administrator can't bring the all photo incorporate information from various partners at one shot. So the clustering figuring which we will realize must be an agglomerative gathering estimation. With this gathering information, an interest document must be manufactured. The chase document will keep up the guide of what features are available at the friend amass as the whole and the whereabouts of each component in the partner cluster[18].

\subsection{Modified Search}

In the request arrange, when the inquiry is sent over P2P sort out, each partner will forward the inquiry to its pack head peer. Once the gathering head peer gets the request, it will look for in the output document and scan for the closeness of the target component proximity or equivalence with any features in its chase record. In case any organizing segment is found the request is occupied to the buddies in which feature is found. If the no organizing area is found, the bundle head will forward to neighbor assemble head[19].

\section{V.CONCLUSION}

The Hybrid part extraction plot for content based picture recovery is proposed in this paper. Half breed include extraction plot which is a mix of shape, shading and textue highlights. For the given Query picture, the low level highlights (shape, shading, and surface) are cleared utilizing distinctive tallies. by then the similarity estimation is performed to picture recovery. The illuminating document contains precisely 1000 subjectively picked pictures for test. Precisely when the outcomes are showed up, the clients have the chance to pass on criticism on every photograph by Relevance input. Our approach constantly increment CBIR execution for the given picture set by developing precision in picture recovery.

\section{REFERENCES}

[1] Wikipedia, -Peer-to-peer, 2011 [Online]. Available: http://en.wikipedia.org/wiki/Peer-to-peer

[2] I. Stoicaet al., -Chord: A scalable peer-to-peer lookup protocol for internet applications, IEEE/ACM Trans. Netw., vol. 11, no. 1, pp. 17-32, Feb. 2003.

[3] X. Li and J. Wu, - Searching techniques in peer-topeer networks, in Handbook of Theoretical and Algorithmic
Aspects of Ad Hoc, Sensor,Peer-to-Peer Networks. Boca Raton, FL, USA: CRC Press, 2004.

[4] C. Gkantsidis, M. Mihail, and A. Saberi, - Random walks in peer-topeer networks, in Proc. IEEE INFOCOM, 2004, pp. 120-130.

[5] C. Gkantsidis, M. Mihail, and A. Saberi, - Hybrid search schemes for unstructured peer to peer networks, in Proc. IEEE INFOCOM, 2005, pp. 1526-1537.

[6] S. Ioannidis and P. Marbach, - On the design of hybrid peer-to-peer systems, in Proc. ACM SIGMETRICS, Annapolis, MD, USA, Jun. 2008, pp. 157-168.

[7] P. Patankar, G. Nam, G. Kesidis, T. Konstantopoulos, and C. Das, -Peer-to-peer unstructured anycasting using correlated swarms, in Proc. ITC, Paris, France, Sep. 2009, pp. 1-8.

[8] R. Gupta and A. Somani, -An incentive driven lookup protocol for chord-based peer-to-peer (P2P) networks, in Proc. Int. Conf. High Perform.Comput., Bangalore, India, Dec. 2004 , pp. $8-18$.

[9] D. Menasche, L. Massoulie, and D. Towsley, - Reciprocity and barter in peer-to-peer systems, in Proc. IEEE INFOCOM, 2010, pp. 1-9.

[10] B. Mitra, A. K. Dubey, S. Ghose, and N. Ganguly, - How do superpeer networks emerge?, in Proc. IEEE INFOCOM, 2010, pp. 1-9.

[11] D. Karger and M. Ruhl, - Simple efficient load balancing algorithms for peer-to-peer systems, in Proc. 16th ACMSPAA, 2004, pp. 36-43.

[12] B. Yang and H. Garcia-Molina, -Designing a superpeer network, in Proc. IEEE ICDE, 2003, pp. 49-60.

[13] L. Tassiulas and A. Ephremides, - Stability properties of constrained queueing systems and scheduling policies for maximum throughput in multihop radio networks, IEEE Trans. Autom. Control, vol. 37, no. 12, pp. 1936-1948, Dec. 1992.

[14] M. J. Neely, E. Modiano, and C. E. Rohrs, -Dynamic power allocation and routing for time varying wireless networks, in Proc. IEEE INFOCOM, 2003, pp. 745755.

[15] L. Ying, S. Shakkottai, A. Reddy, and S. Liu, -On combining shortest-path and back-pressure routing over multihop wireless networks, IEEE/ACM Trans. Netw., vol. 19 , no. 3 , pp. 841-854, Jun. 2011.

[16] M. Alresaini, M. Sathiamoorthy, B. Krishnamachari, and M. Neely, -Backpressure with adaptive redundancy (BWAR), in Proc. IEEE INFOCOM, Mar. 2012, pp. 2300-2308.

[17] L. Bui, R. Srikant, and A. Stolyar, -A novel architecture for reduction of delay and queueing structure complexity in the back-pressure algorithm, IEEE/ACM Trans. Netw., vol. 19, no. 6, pp. 1597-1609, Dec. 2011.

[18] B. Ji, C. Joo, and N. Shroff, -Delay-based backpressure scheduling in multihop wireless networks, IEEE/ACM Trans. Netw., vol. 21, no. 5, pp. 1539-1552, Oct. 2013.

[19] Y. Cui, E. Yeh, and R. Liu, -Enhancing the delay performance of dynamic backpressure algorithms, IEEE/ACM Trans. Netw., 2015, to be published.

[20] L. Georgiadis, M. J. Neely, and L. Tassiulas, —Resource allocation and cross-layer control in wireless networks, Found. Trends Netw., vol. 1, no. 1, pp. 1- 144, 2006 